\title{
Erratum: A New Protocol to Secure AODV in Mobile AdHoc Networks
}

\author{
Avinash Krishnan, Aishwarya Manjunath, and Geetha J. Reddy \\ Department of Computer Science and Engineering \\ M. S. Ramaiah Institute of Technology \\ \{avinash.krishnan, aishwarya.m\}@netapp.com, geetha.y.j@gmail.com \\ http: //www.msrit.edu
}

N. Meghanathan et al. (Eds.): CCSIT 2011, Part III, CCIS 133, pp. 378-389, 2011.

(C) Springer-Verlag Berlin Heidelberg 2011

DOI 10.1007/ 978-3-642-17881-8_46

The paper "A New Protocol to Secure AODV in Mobile AdHoc Networks" appearing on pages 378-389 of this publication has been retracted due to a severe case of plagiarism. 